\title{
Competitividad de las MIPYMES que se dedican a la producción y comercialización de productos lácteos, $2014^{1}$
}

\author{
Meyling Massiel Matamoros Cornejo ${ }^{2}$ \\ Gema Aracely Briones Amador ${ }^{3}$ \\ Josué Efraín Quezada Gámez ${ }^{4}$ \\ Beverly Castillo Herrera ${ }^{5}$
}

\section{RESUMEN}

Esta investigación analiza la competitividad entre las MIPYMES de lacteos situadas en la ciudad de Estelí durante el año 2014, para proponer estrategias que mejoren la capacidad competitiva de estas microempresas. La hipótesis tiene como variable independiente, comercializacion y como variable dependiente, competitividad. Esta investigación es aplicada y cuantitativa; el universo de estudio son 12 MIPYMES de lacteos y 110 distribuidores de estas microempresas. El muestreo es probabilístico aleatorio simple. Se aplicaron encuestas y entrevistas semi estructuradas a propietarios de los negocios y a sus intermediarios. Esta investigación precisa tres ejes teóricos: Competitividad, Comercializacion y los Factores que influyen en la competitividad.

Palabras claves: Competitividad, Comercializacion, MIPYMES, Estelí.

Recibido: 15 de diciembre de 2014

Aceptado: 02 de octubre de 2015

1 Este artículo se basa en la investigación titulada Competitividad de las MIPYMES que se dedican a la producción y comercialización de productos lácteos en la ciudad de Estelí, Año 2014. para optar al título de Administración de Empresas en el año 2014 por la UNAN-Managua, FAREM-Estelí.

2 UNAN-Managua, FAREM-Estelí. Correo electrónico:meymatamoro@yahoo.com

3 UNAN-Managua, FAREM-Estelí. Correo electrónico:gabriones18@yahoo.com

4 UNAN-Managua, FAREM-Estelí. Correo electrónico:josueqgamez@gmail.com

5 Master en Ciencias Sociales por la Universidad de Guadalajara, estudiante del Doctorado en Ciencias Sociales de la Universidad del Zulia. Docente titular de la UNAN-Managua, FAREM-Estelí. Correo electrónico:

beverly.castillo@yahoo.com 


\section{Competitiveness of the MIPYMES engaged in the production and marketing of dairy products, 2014}

\section{SUMARY}

This research analyzes the competitiveness between the MIPYMES of dairy products, located in the city of Estelí in 2014, to suggest strategies to improve the competitiveness of these enterprises. The hypothesis as independent variable is the commercialization and the dependent variable is the competitiveness. This research is applied and quantitative; the study universe is 12 MIPYMES of dairy products and 110 distributors of these microenterprises. The sampling is Simple random probability. Surveys and semi -structured interviews were applied to business owners and their agents. This research requires three theoretical pillars: Competitiveness, commercialization and factors influencing in the competitiveness.

Keywords: Competitiveness, Commercialization, MIPYMES, Estelí. 


\section{INTRODUCCIÓN}

Las MIPYMES en Nicaragua representan el 98\% del universo empresarial, aportan cerca del $40 \%$ del PIB, $60 \%$ de la generación de empleo, y representan el 40\% de una exportación en su totalidad ${ }^{1}$.

La producción de leche y sus derivados son un importante factor de empleo y de obtención de ingresos, especialmente en sectores rurales, y es un componente de primera necesidad en la canasta de consumo familiar.

La competitividad es muy importante para las MIPYMES de productos lácteos ya que les permite desarrollarse y a la vez posesionarse de una gran parte del mercado local, acostumbrado tradicionalmente al consumo de lácteos frescos, lo que les ha permitidos sostenerse en el tiempo y crecer continuamente para responder a las exigencias de la competitividad con productos industrializados de marcas nacionales e internacionales, además de enfrentar desafíos externos e internos tales como la baja producción de leche, los problemas climáticos y la baja tecnología utilizada en los procesos de producción.

En la ciudad de Estelí, uno de los principales problemas al que se enfrentan las MIPYMES de productos lácteos artesanales es la competencia entre las pequeñas empresas informales (cuajada campesina tradicional) que existen en la ciudad y otras empresas que traen sus, desde Chontales y Jinotega. A si mismo inciden en el mercado local los productos industrializados de las empresas: Parmalat, Centrolac y Eskimo,

1 El Presidente del Consejo de la Micro, Pequeña y Mediana Empresa, CONIMIPYME declaro que las MIPYMES en Nicaragua representan el 98\% del universo empresarial“. (Alcocer Gilberto. Entrevista de internet. Fecha: 30 de abril 2014). https:// www.google.com.ni/?gws_rd=cr\&ei=ulqCU8HNEq-2sATZkIHg $\mathrm{Bg} \# \mathrm{q}=$ Entrevistas $+\mathrm{a}+$ Gilberto + Alcocer ++ Presidente + del + Conse jo + de + la + Micro $\% 2 C$.
Los consumidores algunas veces sustituyen los productos lacteos por los productos industriales porque estas implementan estrategias planificadas como: la publicidad, variedad de productos, presentación y registro sanitario.

Por otra parte, el procesamiento y comercialización de los productos lacteos se ven afectadas por la poca producción de leche en temporadas de verano, lo que causa bajos niveles de venta y que los propietarios reduzcan su producción y personal.

No existe motivación e incentivo para los productores de leche, para la entrega de un producto de calidad, pues no se aplica en el acopio una diferenciación de precio por calidad, por parte de los acopiadores de leche locales de estas empresas artesanales formales, en cambio las empresas industriales como: Parmalat, Centrolac y Eskimo premian la calidad de la materia prima.

Esto sigue y seguirá siendo un tema relevante para los productores, porque el empresario MIPYMES del sector lácteo presta poca atención a la calidad de la materia prima con la que trabaja.

Existen normativas de calidad definidas por el MINSA que permite que las empresas sean más competitivas, deben utilizar un Sistema de pasteurización que eliminen todos los organismos patógenos que se puedan expandir rápidamente en la leche, y sin embargo la mayoría no utilizan este sistema y sus productos son de baja calidad y poco competitivos, además no pueden obtener el registro sanitario y solo pueden adquirir una licencia sanitaria que les permite establecerse en el Mercado de manera limitada.

Las limitantes que se presentan en las empresas localizadas en Estelí en el sector lácteo principalmente son de carácter de infraestructura, básicamente por la carencia de recursos de inversión para la adquisición de tecnología en equipos y conocimientos. 
Su gran debilidad se centra en el acceso a financiamiento, para mejorar sus negocios, elevar sus ventas y su competitividad en el Mercado. La banca comercial no financia al sector MIPYMES (incluye el sector lácteos) por falta de garantías sólidas, el financiamiento en la empresas investigadas es nulo.

La mayoría de las MIPYMES de lácteos situadas en la ciudad de Estelí no cuentan con un sistema contable que les permita llevar un mejor control de los ingresos y egresos de sus operaciones, no todas están bien organizadas y muchas trabajan en sus propias casas, no existe una división adecuada de las diferentes áreas en que se desarrolla la empresa, esto dificulta muchas veces el proceso gerencial eficiente, para alcanzar su desarrollo empresarial.

Esta investigación se realizó con la finalidad de conocer la productividad, calidad y servicio de los productos de las MIPYMES del sector lacteos en Estelí y brindar a sus propietarios información y sugerencias, que les permitan tener una visión más clara sobre la realidad que enfrentan sus procesadoras y las necesidades de cambio que enfrentan para poder ser competitivos en el mercado local. Así mismo, les permitirá tomar decisiones que mejoren los resultados para asegurar la rentabilidad y el crecimiento empresarial.

\section{MATERIALES Y MÉTODOS}

La hipótesis de esta investigación expresa: El bajo nivel de comercialización de productos lácteos artesanales está influenciado por la poca capacidad competitiva de las empresas de productos lácteos de la ciudad de Estelí.

Esta es una investigación cuantitativa porque se analiza la relación entre las variables proceso de comercializacion y competitividad de las MIPYMES.

Esta investigación es aplicada por que se enfoca en el estudio de la competitividad entre las MIPYMES de lacteos de la ciudad de Estelí, y se proponen soluciones que permitan mejorar el proceso de producción y comercialización.

Las técnicas de recolección de datos utilizadas fueron: encuestas y entrevistas semi estructuradas dirigidas a propietarios de las empresas de lacteos y a sus distribuidores.

\section{RESULTADOS Y DISCUSIÓN}

\section{Los ejes teóricos de la investigación}

Para tener un soporte teórico, se elaboró el marco teórico-conceptual de investigación en base a: Competitividad, Factores que influyen en la competitividad y comercialización.

La competitividad es la capacidad que tiene una organización de mantener sistemáticamente ventajas competitivas que le permitan alcanzar, sostener y mejorar una determinada posición en el mercado. Más aun, se puede decir que es hacer las cosas mejor que los demás de esta manera la competitividad es directamente relacionada con la forma de planear y desarrollar cualquier iniciativa de negocio. (Porter, $\mathrm{M}$. 2005, pag.225).

\section{Factores que influyen en la competitividad (Kristelle, G. 2013, pag.203).}

Calidad del Producto: es la capacidad de producir satisfactores (sean bien económico o bienes y servicios) que satisfagan las expectativas y las necesidades de los usuarios. Por otro lado, también significa realizar correctamente cada paso del proceso de producción para satisfacer a los clientes internos de la organización y evitar bienes defectuosos. Su importancia se basa en que la satisfacción del cliente aumenta su fidelidad al producto (en organizaciones mercantiles). 
Calidad del servicio: la calidad del servicio está relacionada con la capacidad de satisfacer a clientes, usuarios o ciudadanos en forma honesta, justa, solidaria y transparente, amable puntual etc. logrando altos grados de satisfacción en sus relaciones con la organización o institución proveedora del servicio.

Productividad: la capacidad de producir mayor cantidad de producto de cierta calidad (sean bienes o servicios) con menos recursos. La productividad depende en alto grado de la tecnología (capital físico) usada y la calidad de la información de los trabajadores (factores del capital humano) como así en países industrializados los empleados pueden producir en promedio mucha mayor cantidad de los bienes gracias a la existencia de maquinaria que mecaniza o automatiza parte de los procesos.

Comercialización: La comercialización es el conjunto de las acciones encaminadas a comercializar productos, bienes o servicios. Las técnicas de comercialización abarcan todos los procedimientos y maneras de trabajar para introducir eficazmente los productos en el sistema de distribución (Guerrero Manuel, 2007, pág. 280).

\section{Características generales del sector lácteo en estelí}

\begin{tabular}{lrr}
\hline \multicolumn{1}{c}{ Edad } & Frecuencia & \% \\
\hline $31-40$ & 2 & 18 \\
$41-50$ & 6 & 55 \\
Más de 51 & 3 & 27 \\
Total & $\mathbf{1 1}$ & $\mathbf{1 0 0}$ \\
\hline
\end{tabular}

Cuadro $\mathbf{N}^{\circ}$ 1. Edad de los propietarios

Universo. 11 Empresas de Lácteos.

Fuente: Datos primarios de encuesta. Junio 2014

El 55\% de los propietarios de las empresas de lácteos tienen edades entre 41 y 50 años, el $27 \%$ son mayores de 51 años, y el 18\% oscila edades entre 31 y 40 años. Estos datos demuestran gente que las Empresarios son mayor con algún nivel de experiencia y conocimientos del negocio, lo que le da mayor fortaleza a estas empresas.

El tiempo de operar las empresas nos muestra que el $45 \%$ de estos negocios tienen entre 6 a 10 años de operar y el 55\% tienen más de 16 años, Estas empresas ya pasaron el periodo de prueba ( 5 años) y están en su etapa de consolidación.

Según la opinión de un procesador de lácteos "nuestros negocio tiene 21 años de estar operando debido a la buena aceptación en el mercado y también que el producto que ofertamos a nuestros consumidores cuentan con las exigencias del consumidor para el grado y aprobación de estos" (Entrevista realizada el día viernes, 20 de junio 2014).

\section{Comercialización}

\begin{tabular}{lrr}
\hline Estrategia de comercializaición & Frecuencia & \% \\
\hline Bajo Precio & 5 & 45 \\
Diversidad del producto & 4 & 36 \\
Calidad & 11 & 100 \\
Presentación & $\mathbf{6}$ & $\mathbf{5 5}$ \\
\hline \multicolumn{2}{c}{ Cuadro No $^{\text {2. Estrategía de comercialización }}$} \\
\multicolumn{2}{c}{ Universo. 11 Empresas de Lácteos. }
\end{tabular}

Fuente: Datos primarios de encuesta. Junio 2014

Las principales estrategias de comercialización de estas pequeñas empresas el, 45\% compiten con precios, el $36 \%$ por la diversidad de su oferta, el 100\% dicen con la calidad del producto y el 55\% mediante la presentación del producto. Se observa la poca diversidad del producto y todos dicen tener calidad, lo cual es relativo, por lo tanto se observa una tendencia de oferta únicamente, sin tomar en consideración la demanda que es la clave del mercadeo.

\section{Calidad del producto}

\begin{tabular}{lcr}
\hline \multicolumn{1}{c}{ Calidad del producto } & Frecuencia & \% \\
\hline Muy Buena & 30 & 27 \\
Buena & 80 & 73 \\
Regular & 0 & 0 \\
Mala & 0 & 0 \\
Total & 110 & 100 \\
\hline \multicolumn{3}{c}{ Cuadro No 3. Valoración de la calidad del producto } \\
\multicolumn{4}{c}{ Eniverso. 110 Distribuidores Lácteos. } \\
\multicolumn{4}{c}{. Datos primarios de encuesta. Octubre 2014 }
\end{tabular}


Al preguntar sobre la calidad del producto la respuesta predominante fue que el producto es bueno con un $72 \%$ y muy bueno con $27 \%$, esto quiere decir que la valoración de la calidad es buena por parte de los distribuidores en el rango de los productos lácteos artesanales, ofertados por los productores.

De acuerdo a la opinión de uno de los distribuidores "La calidad de este producto es muy buena ya que tiene un buen sabor y buena presentación y satisface a nuestros clientes" (Entrevista realizada el día viernes 7 de noviembre del 2014).

\section{Calidad del servicio}

\begin{tabular}{lrr}
\hline \multicolumn{1}{c}{ Calidad del servicio } & Frecuencia & \multicolumn{1}{c}{$\%$} \\
\hline Excelente & 40 & 36 \\
Bueno & 70 & 64 \\
Regular & 0 & 0 \\
Total & $\mathbf{1 1 0}$ & $\mathbf{1 0 0}$ \\
\hline
\end{tabular}

Cuadro $\mathrm{N}^{\circ}$ 4. Valoración de la calidad del servicio

Universo. 110 Distribuidores Lácteos.

Fuente: Datos primarios de encuesta. Octubre 2014

Al momento de preguntarle a los distribuidores sobre la valoración con respecto a la calidad del servicio obtuvimos las siguientes respuestas, el $64 \%$ considera la calidad del servicio como muy bueno, 36\% la considera excelente esto nos indica que los productores le brindan un buen servicio a sus intermediarios y tratan de mantenerlos satisfechos para poder cumplir con las necesidades de estos.

\section{Competitividad}

\begin{tabular}{lrr}
\hline \multicolumn{1}{c}{ Competencia } & Frecuencia & \% \\
\hline Lac. Quesito & 10 & 91 \\
Lac. Loza & 9 & 82 \\
Lac. Miraflor & 7 & 64 \\
Lac. Mendoza & 8 & 73 \\
Lac. Del Valle & 5 & 45 \\
Lac. Chavarría & 5 & 45 \\
\hline
\end{tabular}

Cuadro $\mathbf{N}^{\circ}$ 5. Principales empresas que consideran su competencia

Universo. 11 empresas de Lácteos.

Fuente: Datos primarios de encuesta. Junio 2014
Cuando valoramos el nivel de competencia entre los empresarios encontramos que los tres primeros lugares en esta actividad, siendo los de más alta calificación El Quesito con 91\%, Lácteos Loza con 82\%, Lácteos Mendoza 73\% siendo estos los que ocupan los tres lugares más destacados, los demás competidores tienen calificaciones menores a $64 \%$.

\section{FODA}

\section{Fortalezas}

1. Se trata de empresas familiares de bajos costos

2. Los productos tienen buena presentación, buen sabor, buen peso, buena calidad.

3. Los niveles de atención a los clientes son excelentes y sistemáticos.

4. La mayor parte de estas empresas tienen local propio, permite ahorro de recursos.

5. El sector lácteo tiene una alta rentabilidad en época de verano e invierno.

6. El producto de estas empresas es objeto de alta demanda en las dos épocas.

7. La ubicación en el mercado de estas empresas les es ventajosa para atender el mercado local.

\section{Oportunidades}

1. El mercado de estos productos es creciente y se relaciona directamente con el crecimiento de la población.

2. Las redes de comercialización tienen alta experiencia en el manejo de este producto históricamente comercializado en el mercado local.

3. La cultura de consumo de estos productos favorecen este tipo de empresas artesanales o semi artesanales.

\section{Debilidades}

1. Son empresas enfocadas en el mercado local que tiene poca exigencia en calidad y esto no permite mejorar la competitividad y mejorar los ingresos

2. La producción de derivados de la leche se baja en temporada de verano, generando baja de ingresos y 
a veces pérdidas a estos productores.

3. Su capacidad productiva y tecnológica es muy artesanal y es poca competitiva con respecto a los productos industrializados tanto nacionales.

4. No tienen estrategias de crecimiento o mejora tecnológica para el futuro lo cual limita su desarrollo y crecimiento.

5. Son empresas que por falta de una visión empresarial no están organizadas entre sí, ni cuentan con un manejo administrativo profesional.

\section{Amenazas}

1. Alta competencia artesanal y también industrial en el mercado de productos lácteos.

2. Las condiciones climáticas no favorecen la producción de materia prima para esta industria en la zona seca del país.

3. La baja tecnología y la poca calificación de estas empresas, les hace cada día más difícil la competencia en el mercado frente al avance de la industria.

4. Las exigencias de este tipo de industria y de los consumidores, son barreras que limitan y tienden a reducir el crecimiento y desarrollo futuro de estas empresas.

\section{Líneas estratégicas que mejoren la competitividad de las MIPYMES de lácteos.}

Establecimiento de un Programa de Organización y Mejora de la Calidad para las Pequeñas Empresas del sector lácteos de Estelí. Donde se va a Estructurar una propuesta de mejora organizativa del sector lácteo, tambien se va a Diseñar un plan de mejora de la calidad de los productos lácteos.

Desarrollar plan de Mejora Tecnológica del Sector Lácteo en Estelí. Realizar inversiones en infraestructura, maquinaria y equipo de tecnología media para estas pequeñas empresas. Aquí se van a capacitar los recursos humanos de estas pequeñas empresas en los procesos productivos de los derivados de la leche en sus diferentes productos. De igual forma se trabajara en la capacitación de los productores lácteos para mejorar la calidad del producto que acopian estas pequeñas empresas.

Desarrollar un Plan de Competitividad del sector lácteo en Estelí donde se obtenga los registros comerciales, sanitarios, código de barra, empaque de las pequeñas empresas del sector lácteo de Estelí. De esta manera, proceder a la patentacion o registro de marca de los diferentes productos que elaboran las empresas del sector lácteo de la ciudad de Estelí. Y así gestionar certificación de los productos lácteos y registro zoosanitario de las pequeñas empresas de Estelí que pueden tener potencial para la exportación.

Desarrollar un Plan de Mercadeo para las Empresas del Sector Lácteo de Estelí. Utilizar los medios de radiales, audiovisuales, escritos y electrónicos para dar a conocer los productos ante los diferentes mercados locales. Haciendo uso de las relaciones públicas mediante la asistencia a eventos, congresos, ferias, encuentros del sector lácteo a fin de darse a conocer y establecer contactos o conexiones que les permitan mercadear sus productos a diferentes niveles. Y de esta manera lograr que los productores mejoren la presentación de sus productos, con nuevas formas de empaque, logos, eslogan, etiqueta, código de barra que permita la diferenciación e identificación de los productos y su calidad.

\section{CONCLUSIONES}

Al realizar el análisis de los resultados se concluyó que:

Los resultados de nuestra investigación nos muestran que la hipótesis planteada sobre los bajos rendimientos de los procesos de comercialización de los productos lácteos artesanales en la Ciudad de Estelí, es motivada por los bajos niveles de calidad del producto, lo cual queda demostrada con los resultados obtenidos en la 
encuesta; donde los productores reconocen que sus ventas son limitadas y de poca rentabilidad debido a sus bajos niveles de calidad, escasez de la materia prima en verano y la condición perecedera del producto, debido al uso de baja tecnología de producción. Todos estos elementos conllevan a que el producto no presente la mejor calidad y su nivel de rentabilidad sea bajo; lo cual está asociado al reducido tamaño de las empresas, que más del $100 \%$ procesan entre 100 litros y 1, 000 Lts de leche diariamente.

En lo referente a la producción y sus rendimientos, se determinó que la productividad de la leche es baja, por la calidad de la misma desde el proceso de ordeño en la finca, los métodos artesanales de procesamiento y las técnicas utilizadas para cuajar que varían mucho entre los productores; lo cual influye directamente en la productividad, la calidad y la rentabilidad de esta empresas artesanales.

Los procesos de comercialización de esta producción son bastante comunes y cotidianos tanto en sus métodos de venta, como en sus canales de comercialización, sus acciones de promoción y publicidad, no brindan valores agregados a la producción, además de no tener las condiciones de competitividad ante los sectores industriales nacionales que también inciden en el mercado local de la Ciudad de Estelí.

En referencia a las estrategias de comercialización encontramos que en estas pequeñas empresas el $45 \%$ tratan de competir con precios, el $36 \%$ por la diversidad de su oferta, el 100\% dicen que lo hacen por la calidad del producto artesanal y el 55\% se basan en la presentación del mismo.

Con respecto a las características de la Oferta de este sector la diversificación de sus productos es limitada y repetitiva lo que no permite acceder a nichos de mercado con mayor capacidad adquisitiva, lo que limita a estas empresas a un mercado recurrente y cotidiano, que no exige gran calidad, pero tampoco ofrece mejores precios a los pequeños empresarios.
El sector considera que sus clientes identifican sus productos, $82 \%$ por marca, el $36 \%$ por precio, el $73 \%$ por calidad y $64 \%$ por el sabor del producto, las dos condiciones fundamentales para rechazar un producto es el exceso de sal (54.55\%) y de suero (45.45\%), así mismo expresan que lo fundamental para el consumidor es el sabor $(63.64 \%)$.

\section{RECOMENDACIONES}

Recomendamos a los propietarios de las MIPYMES, lo siguiente:

- El sector lácteo artesanal de la Ciudad de Estelí, debe de organizarse, mejorar tecnológicamente, para influir en la calidad y la capacidad competitiva de sus productos.

- Es urgente que este sector, mejore sus niveles tecnológicos de acopio de materia prima y procesamiento de sus productos, para mejorar la calidad del producto.

- El sector lácteo artesanal de Estelí, debe mejorar sus niveles de planeación, organización, dirección y control de sus unidades de producción, para mejorar sus resultados económicos y financieros.

Recomendamos a la Universidad y al departamento de ciencias económicas y empresariales de la debería de dirigir más a los estudiantes a trabajar en los aspectos administrativos, contables, financieros y de mercadeo, con el sector MIPYMES ARTESANAL, que es generador de empleo, producción y movimiento económico, además de estar relacionado directamente con la producción agropecuaria del país.

\section{BIBLIOGRAFÍA}

El Presidente del Consejo de la Micro, Pequeña y Mediana Empresa, CONIMIPYME declaro que las Mipymes en Nicaragua representan el 98\% del universo empresarial”. (Alcocer Gilberto, entrevista tomada de internet el 30 de abril 2014). https://www. google.com.ni/?gws_rd=cr\&ei=ulqCU8HNEq- 
2sATZkIHgBg\#q $=$ Entrevistas $+\mathrm{a}+$ Gilberto + Alcoc er ++ Presidente + del + Consejo + de + la + Micro $\% 2 \mathrm{C}$.

Porter, M. (2005) Porter y la competitividad [versión electrónica] consultado el 16 de abril de 2014. Disponible en: http://sangrefria.com/ blog/2005/02/09poter-y-la-competitividad/

Kristelle, G. (2013) Competitividad [versión electrónica] Consultado el 17 de abril de 2014. Disponible en http//: www.gerencie.com/ competitividad.html
Kotler P. (1996). Dirección de Mercadotecnia. Octava edición, consultado el 22 de abril del 2014. Pág. $224-225$.

Moreno Moreno Karolina, Picado Juárez Arlen, Rodríguez Solórzano. 2007. Caracterización de las empresas procesadoras de productos lacteos de la ciudad de Estelí. Facultad Regional Multidisciplinaria del Norte, FAREM-ESTELI. Pág. 47. 